\title{
Metabolomic Profiling and Toxicokinetics Modeling to Assess the Effects of the Pharmaceutical Diclofenac in the Aquatic Invertebrate Hyalella azteca
}

Qiuguo Fu,* Andreas Scheidegger, Endre Laczko, and Juliane Hollender

Cite This: Environ. Sci. Technol. 2021, 55, 7920-7929

Read Online

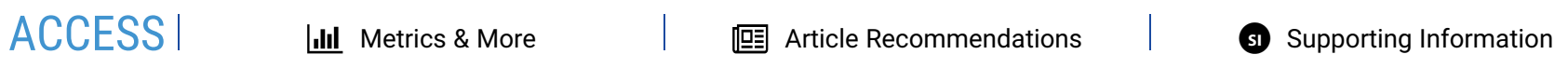

ABSTRACT: The exposure of ecologically critical invertebrate species to biologically active pharmaceuticals poses a serious risk to the aquatic ecosystem. Yet, the fate and toxic effects of pharmaceuticals on these nontarget aquatic invertebrates and the underlying mechanisms are poorly studied. Herein, we investigated the toxicokinetic (TK) processes (i.e., uptake, biotransformation, and elimination) of the pharmaceutical diclofenac and its biotransformation in the freshwater invertebrate Hyalella azteca. We further employed mass spectrometry-based metabolomics to assess the toxic effects of diclofenac on the metabolic functions of

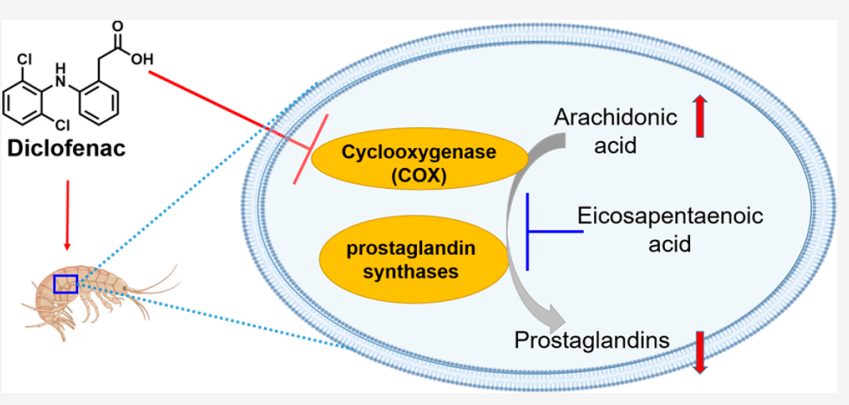
$H$. azteca exposed to environmentally relevant concentrations (10 and $100 \mu \mathrm{g} / \mathrm{L}$ ). The TK results showed a quick uptake of diclofenac by H. azteca (maximum internal concentration of $1.9 \mu \mathrm{mol} / \mathrm{kg}$ ) and rapid formation of the conjugate diclofenac taurine (maximum internal concentration of $80.6 \mu \mathrm{mol} / \mathrm{kg}$ ), indicating over 40 times higher accumulation of diclofenac taurine than that of diclofenac in H. azteca. Depuration kinetics demonstrated that the elimination of diclofenac taurine was 64 times slower than diclofenac in H. azteca. Metabolomics results suggested that diclofenac inhibited prostaglandin synthesis and affected the carnitine shuttle pathway at environmentally relevant concentrations. These findings shed light on the significance of the TK process of diclofenac, especially the formation of diclofenac taurine, as well as the sublethal effects of diclofenac on the bulk metabolome of $H$. azteca. Combining the TK processes and metabolomics provides complementary insights and thus a better mechanistic understanding of the effects of diclofenac in aquatic invertebrates.

KEYWORDS: diclofenac, metabolomics, toxicokinetics, biotransformation, invertebrates, LC-HRMS/MS

\section{INTRODUCTION}

Pharmaceutical pollution has raised global concerns for human health and the environment. Pharmaceutical production and consumption is expected to increase over the coming years driven by a number of factors such as population growth and growing demand for drugs that treat age-related and chronic illness. ${ }^{-3}$ The main classes of pharmaceuticals being produced and used globally are antibiotics, beta-blockers, antidepressants, antiepileptic, contraceptives, lipid regulators, analgesics, and nonsteroidal anti-inflammatory drug (NSAID). ${ }^{3,4}$ These chemicals are continuously being released to the aquatic environment mainly from disposal and excretion of wastewater from household, hospital, and industrial effluent and incomplete removal in the wastewater treatment plants. ${ }^{5-7}$ Once in the aquatic environment, these chemicals may be taken up by the aquatic organisms. Not surprisingly, studies have shown that many pharmaceuticals have been detected in rivers $(\mathrm{ng} / \mathrm{L}-\mu \mathrm{g} /$ L), ${ }^{7,8}$ aquatic vertebrates (e.g., fish), ${ }^{9}$ and invertebrates (e.g., gammarids) (ng/g- $/ \mathrm{g} / \mathrm{g}) .^{10-12}$ Diclofenac as a widely used NSAID in both humans and livestock, has been widely found in various environmental media, including in the $\mathrm{ng} / \mathrm{L}$ to $\mu \mathrm{g} / \mathrm{L}$ range in surface waters and $\mathrm{ng} / \mathrm{g}$ to $\mu \mathrm{g} / \mathrm{g}$ range in aquatic organisms. ${ }^{10,13,14}$ Even though the detected concentrations are low, pharmaceuticals may pose a risk to the nontarget organisms in the aquatic ecosystems because they are designed to be biologically active at low doses for targeted organisms (e.g., humans) and often have a specific mechanism of action (MoA). For example, the pharmacological MoA of diclofenac in humans is the reduction of prostaglandin synthesis by inhibiting cyclooxygenase- 1 (COX-1) and COX-2. ${ }^{15,16}$ Interestingly, a few studies have shown that diclofenac also affects the prostaglandin metabolome in several nontarget organisms such as rainbow trout (Oncorhynchus mykiss), ${ }^{17}$ zebrafish (Danio rerio), ${ }^{18}$ and marine mussels (Mytilus galloprovincialis) by using metabolomics. ${ }^{19,20}$ Also, studies have shown that exposure of diclofenac induces adverse effects in different

Received: November 21, 2020

Revised: May 17, 2021

Accepted: May 17, 2021

Published: June 4, 2021

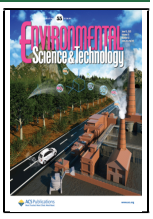


aquatic vertebrates and invertebrates, ${ }^{17,19,21-27}$ as exemplified by the toxic effects in different organs (e.g., cytological alterations in the liver, kidney, and gills) of rainbow trout exposed to environmentally relevant concentrations $(1-5 \mu \mathrm{g} /$ L). ${ }^{21}$

The toxic effects of pharmaceuticals are mainly driven by their toxicokinetic (TK) processes in the living organism. In general, the TK processes include the uptake, biotransformation, distribution, and elimination of the pharmaceuticals and their biotransformation products (BTPs). The uptake process determines how much and how fast a pharmaceutical from the external environment enters the organisms. Then, a pharmaceutical's internal concentration can be eliminated by forming BTPs and/or the excretion via gills, dermal tissue, urine, or feces. Biotransformation is often a vital pathway to detoxify chemicals, but sometimes leads to higher toxicity in biological systems. ${ }^{28-30}$ Thus, biotransformation plays an essential role in influencing pharmaceuticals' TK processes in living organisms. Despite its importance in evaluating overall body burden and the resulting toxicity of diclofenac, studies on its TK processes including biotransformation are rather limited in freshwater invertebrates, who play an essential ecological role in aquatic ecosystems. ${ }^{31}$ There are only a few TK studies of diclofenac in nontarget aquatic organisms, including Gammarus pulex, ${ }^{32}$ oligochaetes Lumbriculus variegatus, ${ }^{33}$ rainbow trout, and zebrafish, ${ }^{34}$ but these studies were only focused on the kinetics of the parent compound diclofenac without considering the biotransformation process. In our recent study, we identified several BTPs of diclofenac, including the two major BTPs diclofenac taurine and diclofenac methyl ester, in aquatic invertebrates Hyalella azteca and Gammarus pulex, but did not investigate their toxicokinetic processes. ${ }^{28}$

TK processes of pharmaceuticals, especially biotransformation, can involve endogenous metabolites (i.e., metabolome) in living organisms for example by enzymatic energy-requiring conjugation with amino acids or sugars. Other TK processes (e.g., bioaccumulation, elimination) can also influence diclofenac's effects on the metabolome by regulating the internal concentrations of diclofenac in organisms. Thus, understanding the metabolome changes induced by pharmaceuticals can help to identify the toxic effects at the molecular level in living organisms. Metabolomics offers a powerful and sensitive technique to characterize metabolome in organisms at the molecular level. ${ }^{35}$ It has been shown that metabolomics is useful to identify the perturbed metabolites and pathways in zebrafish exposed to three pharmaceuticals (i.e., clarithromycin, florfenicol, sulfamethazine, and their mixture) for $72 \mathrm{~h}$ at $100 \mu \mathrm{g} / \mathrm{L}$, at which no observable morphological changes were induced. ${ }^{36}$ Similarly, the prostaglandin metabolome in the common roach (Rutilus rutilus) was dramatically changed by wastewater effluent. ${ }^{37}$ In addition, nontargeted metabolomics could help discover new early response metabolic biomarkers for reproductive impairment of Daphnia magna exposed to cadmium, 2,4-dinitrophenol, or propranolol. ${ }^{38}$ Together, these studies suggest that metabolomics is an appropriate approach to study the toxic effects of diclofenac at the molecular level, which is underexplored for freshwater invertebrates but critical for a comprehensive evaluation of its ecotoxicological risk.

The objective of this study was to evaluate the TK processes of diclofenac and its sublethal effects on the metabolome in Hyalella azteca, a keystone invertebrate species of the aquatic food web. We hypothesized that diclofenac would dysregulate metabolic processes at environmentally relevant concentrations such as the prostaglandin synthesis. Further, the conjugation of diclofenac with endogenous metabolites (i.e., taurine) may influence the metabolome homeostasis of the exposed organisms. To test these hypotheses, first, we monitored the uptake and depuration kinetics of diclofenac and its major conjugate diclofenac taurine in $H$. azteca. Second, we applied TK modeling to quantify the accumulation and elimination rates of diclofenac and diclofenac taurine in $H$. azteca. Third, we profiled the polar and nonpolar metabolome including taurine of $H$. azteca and further identified the perturbed metabolic pathways.

\section{MATERIALS AND METHODS}

Chemicals and Materials. Chemicals and solutions used in this study are provided in the Supporting Information (SI) part $\mathrm{A}$ and Tables S1 and S2. H. azteca were cultured in aerated Borgmann water $(\mathrm{BW})$ at optimal culture conditions $\left(23 \pm 2{ }^{\circ} \mathrm{C}\right.$ and $16 \mathrm{~h} / 8 \mathrm{~h}$ light/dark cycle), with more details on experiments and culture maintenance provided in SI part B.

Exposure Experiments. For the TK exposure experiments, $H$. azteca ( $n=30$, two replicates) were introduced into $600 \mathrm{~mL}$ glass beakers filled with $500 \mathrm{~mL}$ BW. A piece of cotton gauze $(6$ $\times 8 \mathrm{~cm}$ ) was added into each beaker for $H$. azteca to perch and hide. H. azteca were fed every second day with $2 \mathrm{~g}$ of fish food (ground TetraMin powder). The TK experiment was separated into a 10 day-uptake phase and a 5 day-depuration phase. In the 10 day uptake phase, an aliquot of $50 \mu \mathrm{L}$ of stock solution of diclofenac sodium in ethanol (i.e., $10.0 \mathrm{~g} / \mathrm{L}$ ) was spiked to the media to yield an initial diclofenac concentration of $1000 \mu \mathrm{g} / \mathrm{L}$. The stock solution and exposure concentration of diclofenac sodium is in the range of solubility of diclofenac sodium $(21 \mathrm{~g} / \mathrm{L}) .^{39}$ The exposure medium was replaced with the same concentration of fresh medium every 5 days. A glass rod was used to gently stir the medium every day to stabilize the oxygen level in the glass beaker. For the 5 day depuration phase, $H$. azteca was pre-exposed to $1000 \mu \mathrm{g} / \mathrm{L}$ of diclofenac for 1 day, quickly rinsed with nanopure water, and then transferred to the clean BW medium to depurate for 5 days. $H$. azteca were sampled at different sampling times during the uptake and depuration phase (SI part C, Table S3).

For the metabolomics experiment, $H$. azteca $(n=10$, ten replicates) was exposed to increasing concentrations of diclofenac $(0,10,100 \mu \mathrm{g} / \mathrm{L})$ in $600 \mathrm{~mL}$-glass beakers for 1 and 10 days. All concentrations used in this study showed no acute toxicity (no mortality) to $H$. azteca.

At sampling points, animals were sieved, quickly rinsed with nanopure water, blotted dry using tissue paper, transferred into 2 $\mathrm{mL}$ centrifuge tubes, weighed, flash-frozen in liquid nitrogen, and stored at $-80{ }^{\circ} \mathrm{C}$ until further analysis. The exposure medium was sampled in $2 \mathrm{~mL}$ LC-glass vials at the beginning $\left(t_{0 \mathrm{~d}}\right)$, after 1 day $\left(t_{1 \mathrm{~d}}\right)$, and after 10 days $\left(t_{10 \mathrm{~d}}\right)$ of the experiment. Different controls were performed in parallel, i.e., organisms (including food and cotton) without diclofenac in BW medium, diclofenac (including food and cotton) in BW medium without organisms, and only diclofenac in pure BW medium without organisms, food, and cotton.

Sample Preparation. For the TK experiment, the collected animals were spiked with $100 \mu \mathrm{L}$ of methanol containing diclofenac- $\mathrm{d}_{4}(100 \mu \mathrm{g} / \mathrm{L}), 500 \mu \mathrm{L}$ of pure methanol, and $300 \mathrm{mg}$ of $1 \mathrm{~mm}$ zirconia/silica beads (BioSpec Products, Inc.). A FastPrep bead beater (MP Biomedicals) was used for sample homogenization and extraction $\left(15 \mathrm{~s}\right.$ at $6 \mathrm{~m} \mathrm{~s}^{-1}$, two cycles, cooling on ice for $5 \mathrm{~min}$ in between). The homogenate was centrifuged $\left(20000 \mathrm{~g} \times 6 \mathrm{~min}, 20^{\circ} \mathrm{C}\right)$ and filtered through 0.45 
$\mu \mathrm{m}$ regenerated cellulose filters (BGB Analytic AG). The filters were washed with $400 \mu \mathrm{L}$ methanol, and the filtrate and the wash solution were combined. The exposure media $(500 \mu \mathrm{L})$ were spiked with $100 \mu \mathrm{L}$ of methanol containing diclofenac- $\mathrm{d}_{4}(100$ $\mu \mathrm{g} / \mathrm{L})$, and $400 \mu \mathrm{L}$ pure methanol, and mixed evenly. The final extracts and media samples were stored at $-20{ }^{\circ} \mathrm{C}$ prior to diclofenac and its BTP analysis.

For the metabolomics experiments, the sample preparation was performed on wet ice. The collected animals in $2 \mathrm{~mL}$ tubes were placed on wet ice and then mixed with $1 \mathrm{~mL}$ precooled (at $-20{ }^{\circ} \mathrm{C}$ overnight $)$ methanol $/ \mathrm{H}_{2} \mathrm{O}(80: 20)$ and precooled 300 $\mathrm{mg}$ of $1 \mathrm{~mm}$ zirconia/silica beads (BioSpec). Extraction and homogenization were carried out with a FastPrep bead beater (MP Biomedicals) in two cycles of $30 \mathrm{~s}$ at $6.5 \mathrm{~m} \mathrm{~s}^{-1}$ (cooling on wet ice for $5 \mathrm{~min}$ in between). The homogenates were incubated for $10 \mathrm{~min}$ on ice and followed by a centrifuge $(10 \mathrm{~min}, 20000 \mathrm{~g}$, $4{ }^{\circ} \mathrm{C}$ ). The supernatant was transferred to a new precooled $2 \mathrm{~mL}$ Eppendorf tube, and the extraction process was repeated once. The supernatant from the two rounds of extractions was combined and stored at $-80^{\circ} \mathrm{C}$ before the metabolome analysis.

Diclofenac and Biotransformation Products Analysis by Reversed Phase LC-HRMS/MS. The extracted samples of the TK experiment were primarily cleaned and enriched with an automated online-solid phase extraction (SPE) method followed by the reversed phase liquid chromatography-high resolution tandem mass spectrometry (LC-HRMS/MS) analysis using a $\mathrm{Q}$ Exactive mass spectrometer, equipped with an electrospray ionization interface (ESI) (Thermo Fisher Scientific Inc.). ${ }^{28,40}$ Briefly, $200 \mu \mathrm{L}$ of the extract from the TK experiment was added to $20 \mathrm{~mL}$ headspace amber glass vials and filled up with $20 \mathrm{~mL}$ of ultrapure water to be injected into online SPE. After online SPE enrichment, the chromatographic separation was achieved on an XBridge C18 column $(3.5 \mu \mathrm{m}$, $2.1 \times 50 \mathrm{~mm}$, Waters) at $30{ }^{\circ} \mathrm{C}$. Water with $0.1 \%$ (vol.) formic acid and methanol with $0.1 \%$ (vol.) formic acid was used as the mobile phases $\mathrm{A}$ and $\mathrm{B}$, respectively. The gradient program (with respect to mobile phase B) was at $0-5 \mathrm{~min}, 13 \% ; 5-20$ min, 13 to $95 \%$; $20-29.5 \mathrm{~min}, 95 \%$ to $13 \%$; and $29.5-35 \mathrm{~min}$, $13 \%$ at a flow rate of $300 \mu \mathrm{L} / \mathrm{min}$. HRMS $/$ MS was used to detect and quantify diclofenac and its BTPs. Full scan acquisition with a resolution of 70000 (at $m / z 200$ ) was conducted in polarity switching mode followed by data-dependent MS/MS scans (five scans at positive mode, and two at negative mode) with a resolution of $17500($ at $m / z 200)$ and an isolation window of 1 $m / z$. Quantification of compounds with the available reference standards was performed with the respective standard calibration using the software TraceFinder v4.1 (Thermo Scientific). Details of the analytical approaches are provided in SI part D, Tables S4 and S5, and in our previous study. ${ }^{41}$

Nontargeted Analysis of Water-Soluble Metabolites by HILIC-UPLC-HRMS/MS. Before polar metabolomics analysis, the metabolomics experiment's extracts were thoroughly vortexed and prepared in the injection solution (90\% acetonitrile, $8.8 \%$ methanol, $0.056 \%$ ammonium hydroxide, 50 $\mathrm{mM}$ ammonium acetate, $\mathrm{pH} 9$ ). Briefly, $100 \mu \mathrm{L}$ aliquot was transferred to a new Eppendorf tube and blown down to complete dryness under a gentle nitrogen stream. The extract was redissolved in $20 \mu \mathrm{L}$ nanopure water and $80 \mu \mathrm{L}$ of injection solution. The mixture was vortexed for $1 \mathrm{~min}$ and centrifuged at $20000 \mathrm{~g}$ for $10 \mathrm{~min}$ at $4{ }^{\circ} \mathrm{C}$ to eliminate any possible remaining particles. After centrifugation, $80 \mu \mathrm{L}$ of the supernatant was transferred to $1.5 \mathrm{~mL}-\mathrm{LC}$ glass vials. The quality control (QC) sample was prepared by mixing $20 \mu \mathrm{L}$ of each sample and run every 10 th sample in the sequence.

Polar metabolites were analyzed by a Waters nanoACQUITY UPLC coupled to a Waters Synapt G2Si QTOF type MS by a nanoelectrospray ionization (nanoESI) source (Waters Inc.). The chromatographic separation was achieved on a Waters BEH Amide column $(1.7 \mu \mathrm{m}, 0.15 \times 130 \mathrm{~mm})$ at $25^{\circ} \mathrm{C}$. The flow rate was $2 \mu \mathrm{L} / \mathrm{min}$, and the injection volume was $1 \mu \mathrm{L}$. Water (containing $0.5 \mathrm{mM}$ ammonium acetate and $0.05 \%$ ammonium hydroxide, $\mathrm{pH} 9$ ) and acetonitrile (containing $5 \%$ water, $0.5 \mathrm{mM}$ ammonium acetate, and $0.05 \%$ ammonium hydroxide) was used as mobile phases $\mathrm{A}$ and $\mathrm{B}$, respectively. The following gradient program (for mobile phase B) was used: $0-10 \mathrm{~min}, 90$ to $50 \%$; $10.0-10.1 \mathrm{~min}, 50$ to $90 \%$; $10.1-16 \mathrm{~min}, 90 \%$ (SI part E, Table S6). Data independent $\mathrm{MS}^{\mathrm{e}}$ full scan acquisition for a mass range of $50-1200 \mathrm{~m} / z$ with a resolution of 20000 (at $\mathrm{m} / z 554$ ) was conducted using negative ESI polarization at $1.8 \mathrm{kV}$ and an isolation window of $1 \mathrm{Da}$. The mixtures of polar metabolite standards including taurine (SI part E, Table S7) were inserted into the analytical sequence every $10-15$ th sample.

Nontargeted Analysis of Lipophilic Metabolites by Reversed Phase UPLC-HRMS/MS. For nonpolar metabolomics analysis, the metabolomics experiment's extracts were thoroughly vortexed and prepared in the injection solution (methanol/ $\mathrm{H}_{2} \mathrm{O}, 1: 1$ ). Briefly, $100 \mu \mathrm{L}$ aliquot of the extracts was transferred to a new Eppendorf tube and diluted to methanol/ $\mathrm{H}_{2} \mathrm{O}(1: 1)$ by adding $100 \mu \mathrm{L}$ of methanol/ $\mathrm{H}_{2} \mathrm{O}$ (1:4). The mixture was vortexed for $1 \mathrm{~min}$ and centrifuged at $20000 \mathrm{~g}$ for 10 min at $25{ }^{\circ} \mathrm{C}$. After centrifugation, $80 \mu \mathrm{L}$ of the supernatant was transferred to $1.5 \mathrm{~mL}-\mathrm{LC}$ glass vials. The QC sample was prepared in a similar way as above and also measured every 10th sample.

Nonpolar metabolites were analyzed by a nanoACQUITY UPLC (Waters Inc.) coupled with a Picoview nanoESI source (NewObjectives Inc.) to a $\mathrm{Q}$ Exactive mass spectrometer (Thermo Fisher Scientific Inc.). The chromatographic separation was achieved on a Waters HSS T3 C18 column $(1.8 \mu \mathrm{m}$, $0.15 \times 30 \mathrm{~mm}$ ) at $25{ }^{\circ} \mathrm{C}$. Water (containing $5 \%$ acetonitrile, 5 $\mathrm{mM}$ ammonium acetate) and isopropanol (containing $10 \%$ acetonitrile, $5 \mathrm{mM}$ ammonium acetate) were used as mobile phases A and B, respectively. The following gradient program (with respect to mobile phase B) was used: $0-10 \mathrm{~min}, 1 \%$ to 99\%; $10.0-15 \mathrm{~min}, 99 \%$; $15.0-16 \mathrm{~min}, 99 \%$ to $1 \%$; $16.0-20.0$ min, $1 \%$ (SI part F, Table S8). The flow rate was $2 \mu \mathrm{L} / \mathrm{min}$, and the injection volume was $1 \mu \mathrm{L}$. HRMS was performed with positive and negative electrospray polarization in separate runs, with spray voltages of +2.6 and $-1.8 \mathrm{kV}$, respectively. Full scan acquisition for a mass range of $80-1200 \mathrm{~m} / z$ with a resolution setting of 70000 was conducted by data-dependent MS/MS scans (top five most abundant ions) with a resolution setting of 17500 and an isolation window of $1 \mathrm{Da}$. The mixtures of nonpolar metabolite standards (SI part F, Table S9) were also run in the sequence every $10-15$ th samples.

Data Processing, Statistical Analysis, and Pathway Analysis of Metabolomics Data. Progenesis QI (Nonlinear Dynamics, Waters) was used for raw data processing (peak picking, retention time alignment, deconvolution, and normalization) to generate a feature matrix containing compound ID, normalized ion intensity, sample ID, and groups. Parameter settings with Progenesis QI are provided in SI part G, Tables S10 and S11. The feature matrix was then imported into MetaboAnalyst 4.0. Data processing and statistics were performed by following the previously reported step-by-step 
protocol. ${ }^{42}$ Prior to further analysis, quality control was performed for all metabolomic data sets (SI part H, Figure $\mathrm{S} 1)$ to ensure the quality of the acquired data. Principal component analysis (PCA) and partial least-squares discriminant analysis (PLS-DA) was used to check the differences in the metabolic composition among the different treatment groups of diclofenac exposure level and time. The PLS-DA model in each analysis was tested to check for overfitting based on permutation testing. ${ }^{43}$ One-way analysis of variance (ANOVA) and the post hoc testing was used for the multiple pairwise comparisons of each metabolite, to identify the significantly altered metabolites (adjusted $p<0.05$ ). To analyze the potential perturbed metabolic pathways, the list of altered metabolites and their $p$ values were uploaded as input in the module of "MS peaks to pathways" in MetaboAnalyst 4.0 by following the protocol. ${ }^{42}$ The significant metabolites in the perturbed metabolic pathways were then structurally identified by matching MS with the databases of Human Metabolome Database (HMDB, https:// hmdb.ca/), the Kyoto Encyclopedia of Genes and Genomes (KEGG) pathway database (www.genome.jp/kegg/), and lipid maps database (https://www.lipidmaps.org/) as well as MS/ MS fragments with MassBank (MoNA, https://massbank.us/) and mzCloud (https://www.mzcloud.org/), and further confirmed with authentic reference standards if available.

Toxicokinetic (TK) Modeling of Diclofenac and Biotransformation Products. A first-order one-compartment TK model was applied to estimate the rate constants for the toxicokinetic processes. The model is described by the following ordinary differential equations (ODEs):

Parent compound diclofenac:

$$
\frac{\mathrm{d} C_{\mathrm{in}, \mathrm{p}}(t)}{\mathrm{d} t}=C_{\mathrm{water}}(t) \cdot k_{\mathrm{u}}-C_{\mathrm{in}, \mathrm{p}}(t) \cdot k_{\mathrm{e}}-C_{\mathrm{in}, \mathrm{p}}(t) \cdot k_{\mathrm{m}}
$$

The formed product of diclofenac taurine:

$$
\frac{\mathrm{d} C_{\mathrm{in}, \mathrm{m}}(t)}{\mathrm{d} t}=C_{\mathrm{in}, \mathrm{p}}(t) \cdot k_{\mathrm{m}}-C_{\mathrm{in}, \mathrm{m}}(t) \cdot k_{\mathrm{em}}
$$

where $C_{\mathrm{in}, \mathrm{p}}(t)$ and $C_{\mathrm{in}, \mathrm{m}}(t)\left[\mu \mathrm{mol} \mathrm{kg}_{\mathrm{wet}}\right.$ weight (ww) $\left.{ }^{-1}\right]$ are the whole body internal concentrations of the parent compound and the major BTP diclofenac taurine in $H$. azteca, respectively. $C_{\text {water }}(t)\left[\mu \mathrm{mol} \mathrm{L}^{-1}\right]$ describes the parent compound's time course in the exposure medium. Measured exposure medium concentrations during the uptake and depuration phase were used as input for $C_{\text {water }}$. Uptake of the parent compound via food, dermal, and respiratory surfaces is described by the uptake rate constant $k_{u}\left[\mathrm{~L} \mathrm{~kg}_{\mathrm{ww}}{ }^{-1} \mathrm{~d}^{-1}\right] \cdot k_{e}\left[\mathrm{~d}^{-1}\right]$ is a lumped parameter for the direct elimination of the parent compound diclofenac via passive (respiratory and dermal surfaces) and active (excretion of feces) processes as well as the biotransformation of the parent compound to other BTPs. $k_{m}$ and $k_{e m}$, are the biotransformation rate constants $\left[\mathrm{d}^{-1}\right]$ and the elimination (including the direct excretion and further biotransformation) rate constants $\left[\mathrm{d}^{-1}\right]$ for the major BTP, respectively.

The rate constants were obtained by maximum likelihood estimation. We assumed that the model describes the mode of a Gamma distribution with a standard deviation that was kept fixed based on the variance of the replicates. The models were implemented in Julia ${ }^{44}$ with the package DifferentialEquations.jl. ${ }^{45}$ The measured internal concentrations of diclofenac and diclofenac taurine in organisms during toxicokinetic experiments are provided in SI part I, Tables S12 and S13.
Profile likelihood ${ }^{47}$ of the parameters of the one-compartment toxicokinetics model was evaluated with details in SI part J.

Elimination half-lives $\left(t_{1 / 2}\right)$ [d] were calculated for the parent compound diclofenac and its BTP diclofenac taurine based on the total elimination:

$t_{1 / 2}$ of parent compound diclofenac:

$$
t_{1 / 2, \mathrm{p}}=\frac{\ln 2}{k_{\mathrm{e}}+k_{\mathrm{m}}}
$$

$t_{1 / 2}$ of diclofenac taurine:

$$
t_{1 / 2, \mathrm{~m}}=\frac{\ln 2}{k_{\mathrm{em}}}
$$

Additionally, a two-compartment TK model was applied. The details about its equations and description are provided in SI part K.

\section{RESULTS AND DISCUSSION}

Toxicokinetics of Diclofenac and its Major Biotransformation Product in $\mathrm{H}$. azteca. To quantitatively compare the TK of diclofenac and its major BTP, their internal concentrations in $H$. azteca were determined during a 10 day uptake phase and a 5 day depuration phase. We primarily focused on the BTP diclofenac taurine in this study because it is a major BTP, with the concentration found substantially higher than that of its parent compound diclofenac in H. azteca after 10 days. No mortality of $H$. azteca was observed during the experiments. The concentrations of diclofenac in the medium were not significantly changed, no diclofenac was found in chemical-free controls and the temperature and $\mathrm{pH}$ were $23 \pm 2$ ${ }^{\circ} \mathrm{C}$ and $8.1 \pm 0.3$, respectively, throughout experiments. No BTPs were detected in the exposure medium above the detection limit $(0.5 \mathrm{ng} / \mathrm{L})$.

In the uptake phase, diclofenac was quickly taken up by $H$. azteca, and the internal concentrations of diclofenac increased to a maximum of $1.9 \mu \mathrm{mol} / \mathrm{kg}(\hat{=} 0.6 \mathrm{mg} / \mathrm{kg})$ at 1 day and plateaued from 2 to 10 days (Figure 1A). Diclofenac taurine was continuously formed from the beginning of the diclofenac exposure and achieved the maximum internal concentration of $80.6 \mu \mathrm{mol} / \mathrm{kg}(\hat{=} 32.5 \mathrm{mg} / \mathrm{kg})$ after 5 days (Figure 1B). In the depuration phase, the levels of diclofenac in $H$. azteca decreased from $1.9 \mu \mathrm{mol} / \mathrm{kg}(\hat{=} 0.6 \mathrm{mg} / \mathrm{kg})$ to negligible levels $(0.003$ $\mu \mathrm{mol} / \mathrm{kg} \triangleq \hat{=} 0.01 \mathrm{mg} / \mathrm{kg}$ ) at the end of the 5 day depuration phase (Figure 1C), representing a greater than 99\% elimination including excretion and biotransformation. In contrast, the elimination (excretion and further biotransformation) of diclofenac taurine was relatively slow. The levels of diclofenac taurine in H. azteca slowly decreased from $33.6 \mu \mathrm{mol} / \mathrm{kg}(\hat{=} 13.5$ $\mathrm{mg} / \mathrm{kg})$ to $22.3 \mu \mathrm{mol} / \mathrm{kg}(\triangleq=9 \mathrm{mg} / \mathrm{kg})$ at the end of the depuration phase (Figure 1D), indicating only $34 \%$ elimination.

To investigate the TK processes of diclofenac, the time-course concentration of diclofenac and diclofenac taurine in $H$. azteca were simultaneously modeled to gain the rate constant of TK processes including uptake $\left(k_{\mathrm{u}}\right)$, biotransformation $\left(k_{\mathrm{m}}\right)$, and elimination $\left(k_{\mathrm{e}}\right)$. To reduce the number of parameters and uncertainties of TK modeling, only the biotransformation rate constant of diclofenac to diclofenac taurine was set $k_{\mathrm{m}}$, whereas biotransformation of the parent compound to other BTPs was lumped to the elimination rate constant parameters $k_{\mathrm{e}}$. We fitted all parameters for uptake (including 1 and 10 days) and 5 day depuration of diclofenac and diclofenac taurine simultaneously 

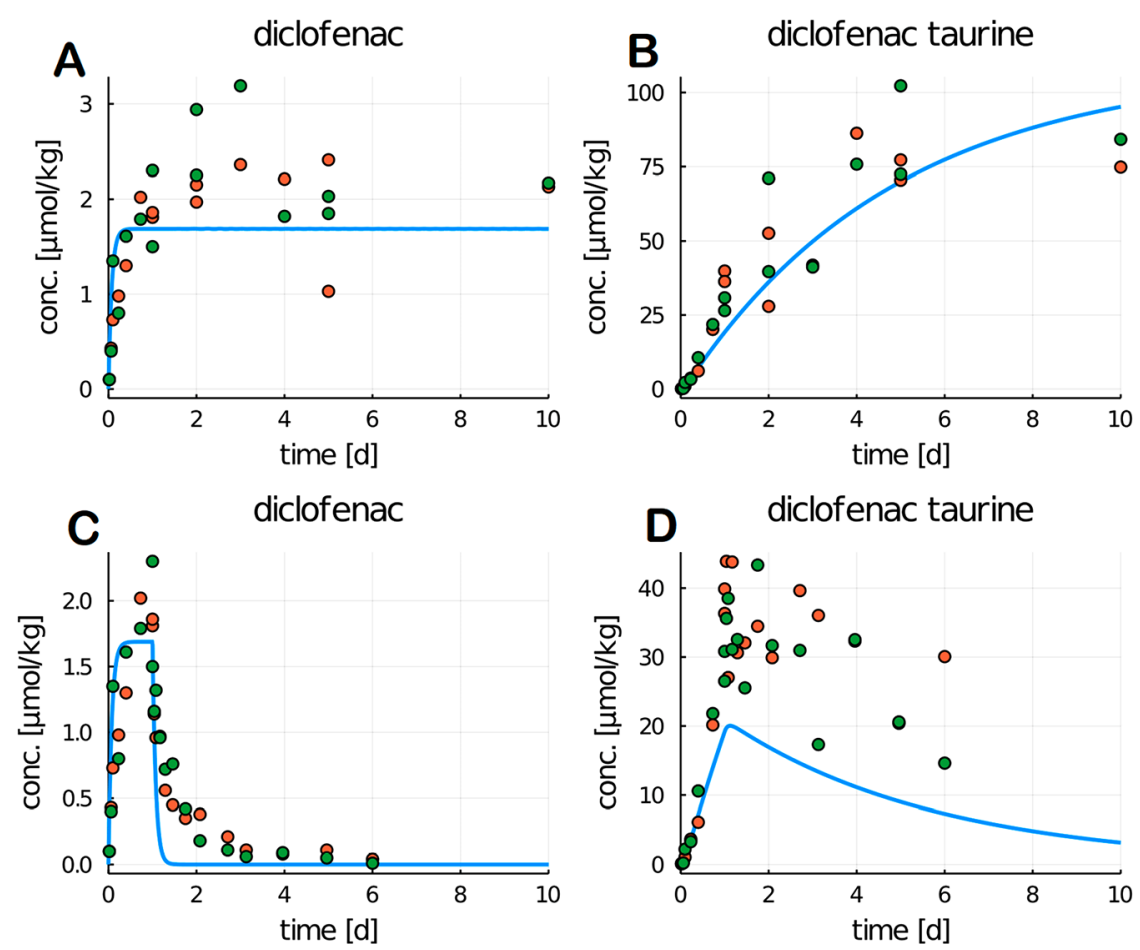

Figure 1. : Toxicokinetics of diclofenac and its BTP diclofenac taurine in H. azteca. (A) The uptake of diclofenac during 10 day exposure (exposure concentration of $1000 \mu \mathrm{g} / \mathrm{L}$ diclofenac). (B) The formation of diclofenac taurine during the 10 day exposure. (C) The kinetics of diclofenac in $H$. azteca during 1 day uptake (diclofenac, $1000 \mu \mathrm{g} / \mathrm{L}$ ) and 5 day depuration (diclofenac, $0 \mu \mathrm{g} / \mathrm{L}$ ). (D) The kinetics of the formed diclofenac taurine in $H$. azteca during 1 day exposure of diclofenac and depuration. Note: depuration started at 1 day uptake. Two different colors represent two replicates at each time point.

because this approach reduces the uncertainty and risk of overfitting. ${ }^{41,46}$

The uptake rate of diclofenac $k_{\mathrm{u}}$ was $6.4 \mathrm{~L} \mathrm{~kg}^{-1} \mathrm{~d}^{-1}$ (95\% CI: 6.30-6.50) in $H$. azteca. The formation rate of diclofenac taurine $k_{\mathrm{m}}$ was $13.5 \mathrm{~d}^{-1}$ (95\% CI: 13.2-13.8). Profile likelihood plots $^{47}$ (SI part I, Figure S2) indicated a very poor identifiability of the elimination rate of diclofenac $k_{\mathrm{e}}$ (including the direct excretion of diclofenac and the biotransformation to other BTPs). While the exact value could not be estimated, it is certainly very small $\left(<1 \times 10^{-10}\right)$. Thus, the total elimination rate $k_{\mathrm{et}}$ of diclofenac $\left(k_{\mathrm{e}}+k_{\mathrm{m}}\right)$ in $H$. azteca can be explained fully $(\sim 100 \%)$ by its transformation to diclofenac taurine. The total elimination rate of diclofenac taurine $\left(k_{\mathrm{em}}\right)$ was $0.21 \mathrm{~d}^{-1}(95 \%$ CI: $0.20-0.22)$ in $H$. azteca, representing 64 times slower elimination of diclofenac taurine compared to $k_{e t}$ of diclofenac. These parameters correspond to the half-life of diclofenac and diclofenac taurine of $0.05 \mathrm{~d}^{-1}$ and $3.3 \mathrm{~d}^{-1}$, respectively. However, the best fit predicts faster elimination of diclofenac and diclofenac taurine during the depuration phase than the experimental data (Figure 1C,D). Thus, these rate constants should be carefully interpreted. Nevertheless, in general, the model was able to describe the experimental data sufficiently well (Figure 1A-D). Furthermore, we tested a two-compartment TK model (SI part K), which provides a better fit (SI part K, Figure S3). However, unique parameter values could not be estimated with the given data (SI part K, Figure S4).

The slower elimination of diclofenac taurine was unexpected because it is more polar $\left(\log D_{\mathrm{ow}}(\mathrm{pH} 7.9), 0.3\right)$ than its parent compound diclofenac $\left(\log D_{\text {ow }}(\mathrm{pH} 7.9), 0.9\right)$, which leads to a lower bioaccumulation prediction. Nevertheless, the slower elimination was also observed for the BTPs of other organic pollutants in $H$. azteca ${ }^{41}$ and other aquatic invertebrates (e.g., gammarids, daphnia). ${ }^{48,49}$ While the exact mechanism is still unclear, Jeon et al. ${ }^{48}$ and Ashauer et al. ${ }^{49}$ speculated that the lower permeability of cell membranes for the hydrophilic (ionized) BTPs leads to ion trapping and decreases the excretion. In mammals (e.g., humans, dogs, mice), the formation of more hydrophilic BTPs via phase II conjugation accounts for a major route of excretion. For example, diclofenac taurine conjugates and its hydroxyl BTPs are mainly excreted via urine and feces in mice and dogs. ${ }^{50,51}$ This may be because mammals have a developed kidney, which contains nephrons that can efficiently filter out the ionized compounds such as taurine conjugate and concentrate them into the urine. However, invertebrates such as $H$. azteca lack this kind of renal function, preventing the excretion of taurine conjugates. Together, these results indicate the relevance of taurine conjugation reactions in aquatic invertebrates.

Influence of the Formation of Diclofenac Taurine on Endogenous Taurine levels and the Other Polar Metabolome in $H$. azteca. To investigate the effects of the formation of diclofenac taurine from diclofenac on the taurine pool in $H$. azteca under environmental settings, we exposed $H$. azteca to more environmentally relevant concentrations (i.e., 10 and $100 \mu \mathrm{g} / \mathrm{L}$ ) of diclofenac. Herein, we determined the internal concentrations of diclofenac, diclofenac taurine, and the endogenous taurine after 1 and 10 day exposure. The internal concentrations of diclofenac were similar between 1 and 10 day exposures, whereas the internal concentration of diclofenac taurine after 10 day exposure was $>3$ times higher than that after 1 day exposure (Figure 2A,B). These results are consistent with the observations in $H$. azteca that were exposed to a higher concentration $(1000 \mu \mathrm{g} / \mathrm{L})$ of diclofenac in the abovementioned TK experiments. The continuous production of 

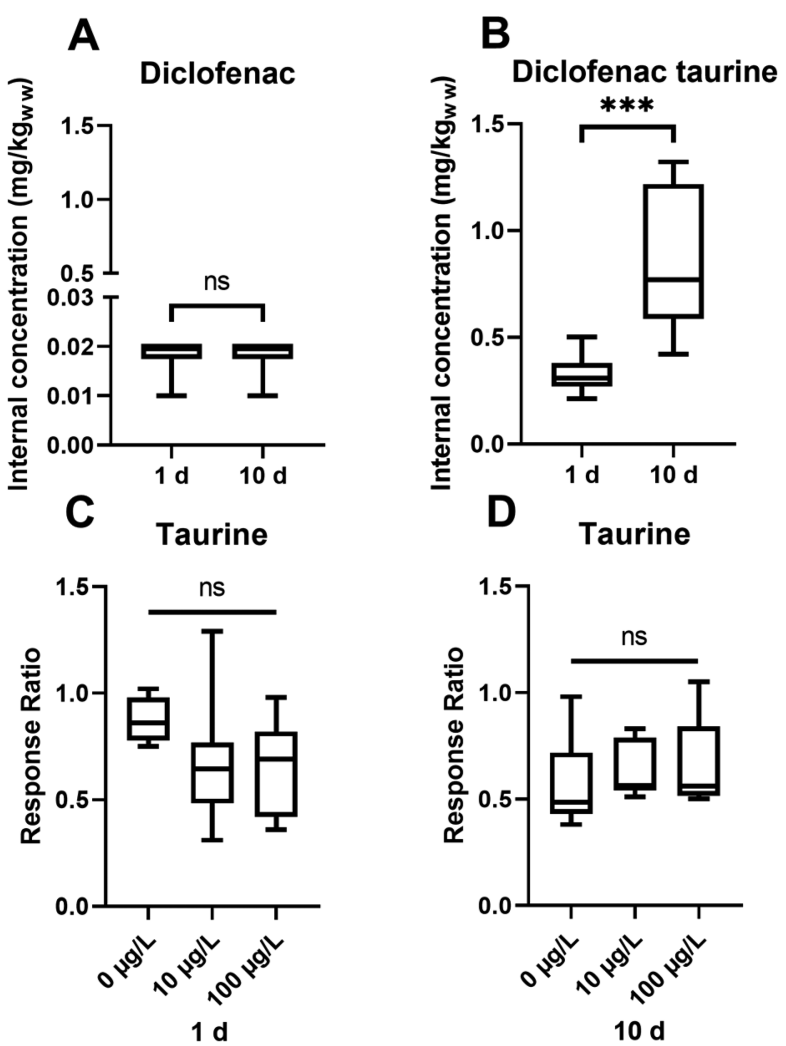

Figure 2. : Accumulation of diclofenac and diclofenac taurine does not directly affect endogenous taurine levels in $H$. azteca. The internal concentration of diclofenac (A) and diclofenac taurine (B) in H. azteca exposed to $100 \mu \mathrm{g} / \mathrm{L}$ (note: internal concentration of diclofenac and diclofenac taurine at $10 \mu \mathrm{g} / \mathrm{L}$ was below detection limit); the response level of taurine (i.e., taurine/taurine- $\mathrm{d}_{4}$ ) in $\mathrm{H}$. azteca exposed to 0,10 , $100 \mu \mathrm{g} / \mathrm{L}$ at 1 day (C) and 10 days (D). Note: diclofenac and diclofenac taurine were analyzed by RP-UPLC-HRMS/MS, whereas taurine was measured by HILIC-UPLC-HRMS/MS. ns stands for not significant. *** stands for significant.

diclofenac taurine requires taurine consumption, an amino acid that is involved in many functions such as bile salt formation and membrane stability. ${ }^{52}$ Thus, we hypothesized that the formation of diclofenac taurine might influence the taurine pool in the tissues of $H$. azteca. We first calculated the ratio of the response area of taurine and that of taurine- $\mathrm{d}_{4}$ in the organisms, and then compared the response ratios among different exposure groups of $H$. azteca. The taurine response ratios among three exposure levels at 1 and 10 day exposure were not significantly different (Figure 2C,D), which was against our hypothesis. These results indicate that diclofenac taurine accumulation did not directly affect the taurine pool in $H$. azteca after 10 days exposure at the sublethal concentration (10 and $100 \mu \mathrm{g} / \mathrm{L})$. Besides, taurine level $(16-25 \mathrm{mg} / \mathrm{kg})$ in exposed $H$. azteca was $>10$ times higher than diclofenac taurine $(0.2-1.3 \mathrm{mg} / \mathrm{kg})$ based on our semiquantification data, suggesting that the conjugation of diclofenac only consumed a limited proportion of the taurine in $H$. azteca and thus did not disrupt the taurine pool.

We further examined the effects of diclofenac on the metabolome of $H$. azteca by nontargeted profiling of polar endogenous metabolites. PCA score modeling showed no separation between the three different exposure concentrations $(0,10$, and $100 \mu \mathrm{g} / \mathrm{L})$ at 1 and 10 day exposure and no significant metabolite was observed after the ANOVA and post hoc testing statistical analysis (SI part L, Figure S5A,B). PLS-DA modeling gave a better separation among the three exposure concentrations at both 1 and 10 day exposure (SI part L, Figure S5C,). Still, the modeling was probably overfitting, given that the Q2 values were less than 0.5 . The overfitting is supported by the results from ANOVA and the post hoc analysis, which showed no significant features $(p>0.05)$ in $H$. azteca after 1 day exposure, and only 24 significantly changed features $(p<0.05)$ after 10 day exposure. These results suggest that diclofenac exposure had a limited effect on polar metabolites including taurine in $H$. azteca. In addition, no significantly perturbed biological pathways were observed based on the polar metabolomics data. At similar or lower concentrations of diclofenac $(0-25 \mu \mathrm{g} / \mathrm{L})$, Mehinto et al. ${ }^{53}$ also found that there is no distinct separation in the PCA and PLS-DA modeling of bile and plasma metabolome of fish. Together, these results suggest that diclofenac at environmentally relevant concentrations (10, $100 \mu \mathrm{g} / \mathrm{L})$ might not significantly impact the core polar metabolic functions in $H$. azteca.

Dose-Dependent Effects of Diclofenac on the Nonpolar Metabolome in $\boldsymbol{H}$. azteca. To further explore the effects of diclofenac on the metabolome in $H$. azteca, extracts of the exposed $H$. azteca $(0,10$, and $100 \mu \mathrm{g} / \mathrm{L})$ were profiled for nonpolar metabolites, that is, lipids by reversed phase UPLCHRMS/MS in both positive and negative modes. PLS-DA modeling of the three data sets revealed a clear separation between the three exposure concentrations after 1 day as well as 10 day exposure and a dose-dependent effect (SI part L, Figure S6). PLS-DA modeling's Q2 values were $>0.8$ for both 1 and 10 day exposure, indicating that the models were not overfitting. ANOVA post hoc analysis suggests that 1123 and 1015 features were significantly changed $(p<0.05)$ in H. azteca after 1 and 10 day exposure, respectively, among the lipids detected using ESI negative mode. Using ESI positive mode, 2113 and 1031 significantly changed $(p<0.05)$ features were detected in $H$. azteca after 1 and 10 day exposure, respectively (SI part L, Figure S7). These results suggest that diclofenac's effects on the nonpolar metabolome in $H$. azteca.

MetaboAnalyst 4.0 was used to further analyze the potential perturbed metabolic pathways. Biological pathways were characterized for the diclofenac-induced metabolic perturbation based on the metabolites that had changed significantly. Among pathways, prostaglandin metabolism and carnitine shuttle pathways were significantly impacted and continuously detected in samples from different analysis modes and exposure lengths, suggesting that they were the most relevant pathways influenced by diclofenac (Figure 3A-D). The perturbed metabolites were identified based on the mass spectrum libraries and/or available reference standards. Arachidic acid, arachidonic acid, eicosapentaenoic acid, prostaglandin E1, o-oleoylcarnitine, hexadecanoylcarnitine/palmitoylcarnitine were further confirmed by their reference standards (SI part M, Table S14). The prostaglandin metabolism involves the cyclooxygenase (COX) and prostaglandin synthases (PGS), which is known to be inhibited by another endogenous metabolite eicosapentaenoic acid or xenobiotic COX inhibitor diclofenac (Figure 3A). Dihomo- $\gamma$-linolenic acid can be converted to arachidonic acid, which can then be converted to prostaglandins by PGS, or to the other two metabolites, namely arachidic acid and 2-arachidonoylglycerol. Prostaglandin E1 was decreased with diclofenac exposure in $H$. azteca and this decrease was not alleviated even when the inhibition of eicosapentaenoic acid was decreased (Figure 3B). These results indicate that the synthesis of prostaglandin $\mathrm{E}$ was inhibited in $H$. azteca. In addition to 

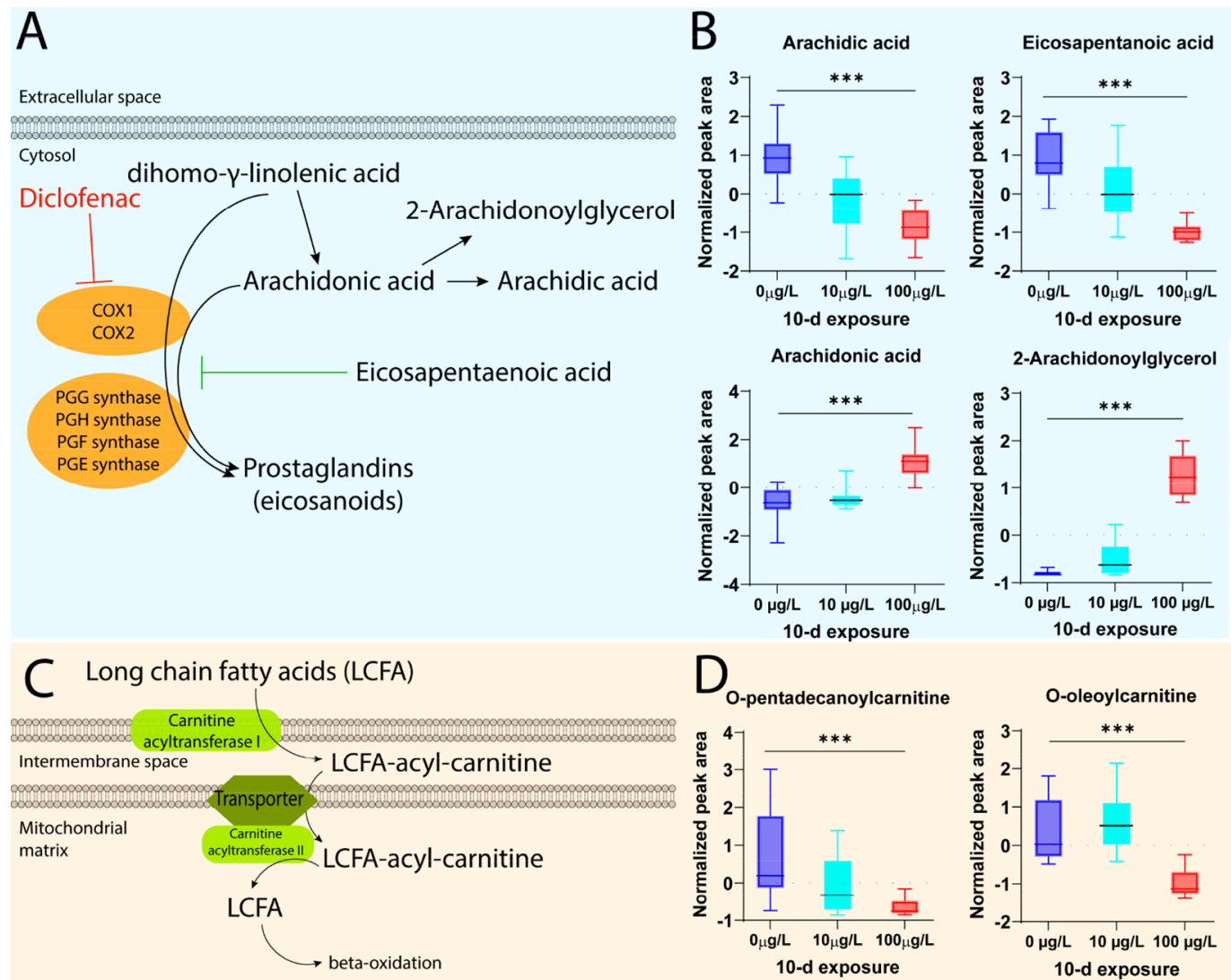

10-d exposure
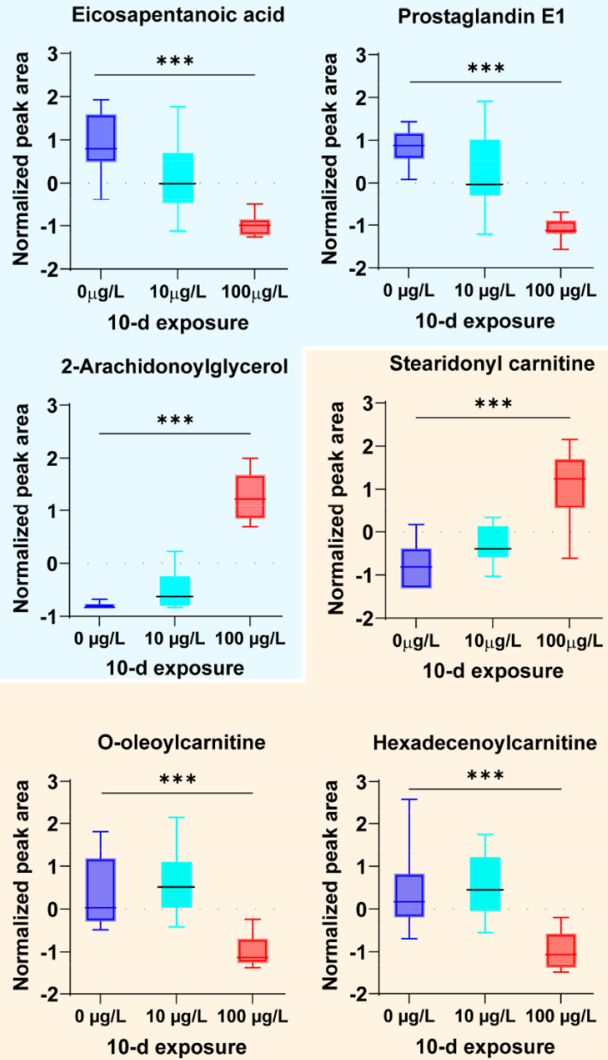

Figure 3. The perturbed prostaglandin and carnitine shuttle metabolism pathways. (A) Perturbation of the prostaglandin metabolism induced by diclofenac. (B) Dose-dependent changes of representative metabolites, that is, arachidic acid, arachidonic acid, eicosapentaenoic acid, prostaglandin E1, and 2- arachidonoylglycerol in prostaglandin metabolism pathways. (C) Perturbation of the carnitine shuttle pathway metabolism induced by diclofenac. (D) Dose-dependent changes of representative metabolites (e.g., stearidonylcarnitine, o-pentadecanoyl carnitine, o-oleoylcarnitine, hexadecanoylcarnitine/palmitoylcarnitine) in the carnitine shuttle pathway. Note: QE- and QE+ in (A) and (B) represent the negative and positive scan mode, respectively, with a Q-Exactive mass spectrometer. Data are presented as the normalized peak area. $N=10$. ANOVA post hoc analysis was used during the statistical analysis in MetaboAnalyst 4.0.***: $p<0.001$. Bars in the box plot indicate the minimum and maximum value, and lines in the boxes indicate the mean and quartiles.

COX inhibition, the decreased level of arachidonic acid upon exposure to diclofenac could also contribute to the reduced level of prostaglandin E. The decrease of arachidonic acid is perhaps because other metabolic pathways consuming arachidonic acid were induced. Indeed, we observed arachidic acid and 2arachidonoylglycerol (two other metabolites of arachidonic acid) were significantly increased by diclofenac in a dosedependent manner (Figure 3B). Similarly, Courant et al. ${ }^{20}$ found a dose-dependent reduction of prostaglandin $\mathrm{E}$ levels in marine mussel after exposure to diclofenac for $72 \mathrm{~h}$ at 0,1 , and $100 \mu \mathrm{g} / \mathrm{L}$. Importantly, the disruption of prostaglandins was also induced by treated wastewater that contained diclofenac and other NSAIDs (e.g., ibuprofen, naproxen, felbinac, mefenamic, and celecoxib), ${ }^{37}$ which are other known COX2 or COX1 inhibitors. Thus, according to our hypothesis, the inhibitory effects of diclofenac on COX was similar in H.azteca as in other aquatic species, including zebrafish, rainbow trout, and marine mussel. ${ }^{17,18,20}$ Interestingly, diclofenac's inhibitory effects on COX in $H$. azteca and other aquatic species share a similar MoA of diclofenac in humans. ${ }^{15,16}$ As prostaglandins are essential signaling molecules in regulating physiology, and these results together highlight the importance of understanding the effects of this perturbation on aquatic organisms.

Diclofenac exposure also significantly affected the carnitine shuttle pathway in $H$. azteca. The carnitine shuttle is a critical process of transporting long-chain fatty acids from cell plasma across the membrane to mitochondria subsequent $\beta$-oxidation (Figure $3 \mathrm{C}$ ). This process is carried out by forming acylcarnitine species and subsequent transport by carnitine transporters. ${ }^{54}$ Among the four identified acylcarnitines, three of them significantly decreased in $H$. azteca after exposure to diclofenac (Figure 3D), indicating the reduced formation of fatty acid acylcarnitines. Similarly, Sepúlveda et al. found that the carnitine shuttle pathway was also affected in $H$. azteca exposed to atrazine by using metabolomics analysis. ${ }^{55}$ The formation of carnitine conjugates of fatty acids is catalyzed by carnitine acyltransferases such as carnitine O-octanoyltransferase (CROT). Ibuprofen, a diclofenac-like NSAID drug, affected the expression of CROT in ibuprofen-treated mice, which in turn could change the fatty acid $\beta$-oxidation and lipid metabolism. ${ }^{56}$ Together, these results suggest that diclofenac and other NSAIDs alter the carnitine shuttle pathway of fatty acids and potentially lipid metabolism, especially the fatty acid $\beta$-oxidation in various species.

Environmental Implications. Biotransformation plays a critical role in regulating the fate of contaminants in organisms. Our findings highlight that biotransformation via conjugation influences the toxicokinetic processes and thus, bioaccumulation of the pharmaceutical diclofenac in the aquatic invertebrate $H$. azteca. The unexpected slower elimination of the polar BTP diclofenac taurine in $H$. azteca suggested the importance of 
toxicokinetic information for both the precursor chemicals and their BTPs. In addition, the slow elimination of diclofenac taurine in the invertebrate $H$. azteca is distinct from the fast excretion of taurine conjugates that have been observed in vertebrates, including humans. This species' difference highlights the necessity of ecotoxicological risk assessment of pharmaceuticals to nontarget invertebrates. The toxicokinetics model can predict the uptake and depuration behavior under different exposure scenarios in the environment. ${ }^{57}$ However, the impact of environmental factors such as temperature, $\mathrm{pH}$, food resources likely influence the rates of uptake and depuration.

Metabolomics analysis in the current study revealed that diclofenac affects the prostaglandin metabolism and carnitine shuttle pathways in $H$. azteca at environmentally relevant concentrations. Our results confirm that the MoA of diclofenac in aquatic invertebrates $H$. azteca, that is, inhibition of prostaglandin biosynthesis, is similar to that in humans, exemplifying the read-across hypothesis. ${ }^{58}$ Notably, the identified carnitine shuttle is a newly identified pathway being altered by diclofenac at environmentally relevant concentrations. Further investigation on the impact of these molecular changes in tissue physiology and eventually reproduction and population of $H$. azteca is needed. Nonetheless, these findings support that metabolomics is a powerful tool to advance our understanding of the endogenous molecular changes and the potentially toxic effects of organic contaminants on nontarget organisms. While it was not investigated in the current study, pharmaceuticals' distribution into specific tissues by mass spectrometry imaging-based metabolomics and multicompartment TK modeling within an organism is recommended since certain pathways may be relevant in specific organs. The exploration of the localized effects will help better understand pharmaceuticals' potential risk and their underlying mechanism of action.

\section{ASSOCIATED CONTENT}

\section{SI Supporting Information}

The Supporting Information is available free of charge at https://pubs.acs.org/doi/10.1021/acs.est.0c07887.

Additional details (Supporting Information and Methods. SI parts A-M; Figures S1-S7; Tables S1-S14) PDF)

Code and data for the one compartment and the twocompartment toxicokinetic models (ZIP)

\section{AUTHOR INFORMATION}

\section{Corresponding Author}

Qiuguo Fu - Eawag, Swiss Federal Institute of Aquatic Science and Technology, 8600 Dübendorf, Switzerland; 이이.org/ 0000-0002-4227-5948; Phone: +41 58765 5945;

Email: qiuguo.fu@eawag.ch; Fax: +41 587655802

\section{Authors}

Andreas Scheidegger - Eawag, Swiss Federal Institute of Aquatic Science and Technology, 8600 Dübendorf, Switzerland

Endre Laczko - Functional Genomics Center Zurich, ETH, University of Zurich, 8057 Zurich, Switzerland

Juliane Hollender - Eawag, Swiss Federal Institute of Aquatic Science and Technology, 8600 Dübendorf, Switzerland; Institute of Biogeochemistry and Pollutant Dynamics, ETH Zürich, 8092 Zürich, Switzerland; 이이.org/0000-00024660-274X
Complete contact information is available at:

https://pubs.acs.org/10.1021/acs.est.0c07887

\section{Notes}

The authors declare no competing financial interest.

\section{ACKNOWLEDGMENTS}

This research received funding from the Swiss National Science Foundation (grant number 205320165935). Thanks to Davide Fedrizzi for his assistance in the toxicokinetics experiment, Sebastian Streb (FGCZ) for help with the UPLC-HRMS/MS analysis. We appreciate Tjalling Jager (DEBtox Research) to support toxicokinetics modeling and programming. We also thank Reynold Chow (Eawag) for the helpful discussion and proofreading of the manuscript.

\section{REFERENCES}

(1) Wang, Z.; Walker, G. W.; Muir, D. C. G.; Nagatani-Yoshida, K. Toward a Global Understanding of Chemical Pollution: A First Comprehensive Analysis of National and Regional Chemical Inventories. Environ. Sci. Technol. 2020, 54 (5), 2575-2584.

(2) Rzymski, P.; Drewek, A.; Klimaszyk, P. Pharmaceutical Pollution of Aquatic Environment: An Emerging and Enormous Challenge. Limnol. Rev. 2017, 17 (2), 97-107.

(3) Monteiro, S. C.; Boxall, A. B. A. Occurrence and Fate of Human Pharmaceuticals in the Environment; 2010; Vol. 202. DOI: 10.1007/ 978-1-4419-1157-5 2.

(4) Fent, K.; Weston, A. A.; Caminada, D. Ecotoxicology of Human Pharmaceuticals. Aquat. Toxicol. 2006, 76 (2), 122-159.

(5) Anliker, S.; Patrick, M.; Fenner, K.; Singer, H. Quantification of Active Ingredient Losses from Formulating Pharmaceutical Industries and Contribution to Wastewater Treatment Plant Emissions. Environ. Sci. Technol. 2020, 54 (23), 15046-15056.

(6) Anliker, S.; Loos, M.; Comte, R.; Ruff, M.; Fenner, K.; Singer, H. Assessing Emissions from Pharmaceutical Manufacturing Based on Temporal High-Resolution Mass Spectrometry Data. Environ. Sci. Technol. 2020, 54 (7), 4110-4120.

(7) Diáz-Cruz, S.; Barceló, D. Occurrence and Analysis of Selected Pharmaceuticals and Metabolites as Contaminants Present in Waste Waters, Sludge and Sediments. Handb. Environ. Chem. 2004, 5, 227260

(8) McEachran, A. D.; Shea, D.; Bodnar, W.; Nichols, E. G. Pharmaceutical Occurrence in Groundwater and Surface Waters in Forests Land-Applied with Municipal Wastewater. Environ. Toxicol. Chem. 2016, 35 (4), 898-905.

(9) Pico, Y.; Belenguer, V.; Corcellas, C.; Diaz-Cruz, M. S.; Eljarrat, E.; Farré, M.; Gago-Ferrero, P.; Huerta, B.; Navarro-Ortega, A.; Petrovic, M.; Rodríguez-Mozaz, S.; Sabater, L.; Santín, G.; Barcelo, D. Contaminants of Emerging Concern in Freshwater Fish from Four Spanish Rivers. Sci. Total Environ. 2019, 659, 1186-1198.

(10) Munz, N. A.; Fu, Q.; Stamm, C.; Hollender, J. Internal Concentrations in Gammarids Reveal Increased Risk of Organic Micropollutants in Wastewater-Impacted Streams. Environ. Sci. Technol. 2018, 52 (18), 10347-10358.

(11) Miller, T. H.; Ng, K. T.; Bury, S. E.; Bury, N. R.; Barron, L. P. Biomonitoring of Pesticides, Pharmaceuticals and Illicit Drugs in a Freshwater Invertebrate to Estimate Toxic or Effect Pressure. Environ. Int. 2019, 129, 595-606.

(12) Shahid, N.; Becker, J. M.; Krauss, M.; Brack, W.; Liess, M. Pesticide Body Burden of the Crustacean Gammarus Pulex as a Measure of Toxic Pressure in Agricultural Streams. Environ. Sci. Technol. 2018, 52 (14), 7823-7832.

(13) Munz, N. A.; Burdon, F. J.; de Zwart, D.; Junghans, M.; Melo, L.; Reyes, M.; Schönenberger, U.; Singer, H. P.; Spycher, B.; Hollender, J.; Stamm, C. Pesticides Drive Risk of Micropollutants in WastewaterImpacted Streams during Low Flow Conditions. Water Res. 2017, 110, 366-377. 
(14) European Union Commission Implementing Regulation (EU) 2015/ 495 of 20 March 2015 Establishing a Watch List of Substances for UnionWide Monitoring in the Field of Water Policy Pursuant to Directive 2008/ 105/EC of the European Parliament and of the Council; 2015; Vol. L78/ 40. http://eur-lex.europa.eu/pri/en/oj/dat/2003/1_285/1_ 28520031101en00330037.pdf.

(15) Kurumbail, R. G.; Stevens, A. M.; Gierse, J. K.; Mcdonald, J. J.; Stegeman, R. A.; Pak, J. Y.; Gildehaus, D.; Miyashiro, J. M.; Penning, T. D.; Seibert, K.; Isakson, P. C.; Stallings, W. C. Structural Basis for Selective Inhibition of Cyclooxygenase-2 by Anti-Inflammatory Agents. Nature 1996, 384, 538-541.

(16) Gan, T. J. Diclofenac: An Update on Its Mechanism of Action and Safety Profile. Curr. Med. Res. Opin. 2010, 26 (7), 1715-1731.

(17) Mehinto, A. C.; Hill, E. M.; Tyler, C. R. Uptake and Biological Effects of Environmentally Relevant Concentrations of the Nonsteroidal Anti-Inflammatory Pharmaceutical Diclofenac in Rainbow Trout (Oncorhynchus Mykiss). Environ. Sci. Technol. 2010, 44 (6), $2176-2182$.

(18) Klüver, N.; Bittermann, K.; Escher, B. I. QSAR for Baseline Toxicity and Classification of Specific Modes of Action of Ionizable Organic Chemicals in the Zebrafish Embryo Toxicity Test. Aquat. Toxicol. 2019, 207 (June 2018), 110-119.

(19) Bonnefille, B.; Gomez, E.; Alali, M.; Rosain, D.; Fenet, H.; Courant, F. Metabolomics Assessment of the Effects of Diclofenac Exposure on Mytilus Galloprovincialis: Potential Effects on Osmoregulation and Reproduction. Sci. Total Environ. 2018, 613-614, 611618.

(20) Courant, F.; Arpin-Pont, L.; Bonnefille, B.; Vacher, S.; PicotGroz, M.; Gomez, E.; Fenet, H. Exposure of Marine Mussels to Diclofenac: Modulation of Prostaglandin Biosynthesis. Environ. Sci. Pollut. Res. 2018, 25 (7), 6087-6094.

(21) Schwaiger, J.; Ferling, H.; Mallow, U.; Wintermayr, H.; Negele, R. D. Toxic Effects of the Non-Steroidal Anti-Inflammatory Drug Diclofenac. Part I: Histopathological Alterations and Bioaccumulation in Rainbow Trout. Aquat. Toxicol. 2004, 68 (2), 141-150.

(22) Gómez-Oliván, L. M.; García-Medina, S.; Galar-Martínez, M.; Islas-Flores, H.; Colín-Cruz, A.; Neri-Cruz, N. Diclofenac-Induced Oxidative Stress in Brain, Liver, Gill and Blood of Common Carp (Cyprinus Carpio). Ecotoxicol. Environ. Saf. 2013, 92, 32-38.

(23) Triebskorn, R.; Casper, H.; Heyd, a; Eikemper, R.; Köhler, H.-R.; Schwaiger, J. Toxic Effects of the Non-Steroidal Anti-Inflammatory Drug Diclofenac. Aquat. Toxicol. 2004, 68 (2), 151-166.

(24) Hoeger, B.; Köllner, B.; Dietrich, D. R.; Hitzfeld, B. Water-Borne Diclofenac Affects Kidney and Gill Integrity and Selected Immune Parameters in Brown Trout (Salmo Trutta f. Fario). Aquat. Toxicol. 2005, 75 (1), 53-64.

(25) Schwarz, S.; Schmieg, H.; Scheurer, M.; Köhler, H. R.; Triebskorn, R. Impact of the NSAID Diclofenac on Survival, Development, Behaviour and Health of Embryonic and Juvenile Stages of Brown Trout, Salmo Trutta f. Fario. Sci. Total Environ. 2017, 607608, 1026-1036.

(26) Norrgren, L.; Näslund, J.; Larsson, D. G. J.; Asker, N.; Fick, J.; Ekman, E. Diclofenac Affects Kidney Histology in the Three-Spined Stickleback (Gasterosteus Aculeatus) at Low $\mathrm{Mg} / \mathrm{L}$ Concentrations. Aquat. Toxicol. 2017, 189 (April), 87-96.

(27) Świacka, K.; Smolarz, K.; Maculewicz, J.; Caban, M. Effects of Environmentally Relevant Concentrations of Diclofenac in Mytilus Trossulus. Sci. Total Environ. 2020, 737. DOI: 10.1016/j.scitotenv.2020.139797.

(28) Fu, Q.; Fedrizzi, D.; Kosfeld, V.; Schlechtriem, C.; Ganz, V.; Derrer, S.; Rentsch, D.; Hollender, J. Biotransformation Changes Bioaccumulation and Toxicity of Diclofenac in Aquatic Organisms. Environ. Sci. Technol. 2020, 54 (7), 4400-4408.

(29) Ashrap, P.; Zheng, G.; Wan, Y.; Li, T.; Hu, W.; Li, W.; Zhang, H.; Zhang, Z.; Hu, J. Discovery of a Widespread Metabolic Pathway within and among Phenolic Xenobiotics. Proc. Natl. Acad. Sci. U. S. A. 2017, 114 (23), 6062-6067.

(30) McCormick, J. M.; Es, T. Van; Cooper, K. R.; White, L. A.; Häggblom, M. M. Microbially Mediated O-Methylation of Bisphenol a
Results in Metabolites with Increased Toxicity to the Developing Zebrafish (Danio Rerio) Embryo. Environ. Sci. Technol. 2011, 45 (15), 6567-6574.

(31) Wallace, J. B.; Webster, J. R. The Role of Macroinvertebrates in Stream Ecosystem Function. Annu. Rev. Entomol. 1996, 41 (1), 115139.

(32) Miller, T. H.; McEneff, G. L.; Stott, L. C.; Owen, S. F.; Bury, N. R.; Barron, L. P. Assessing the Reliability of Uptake and Elimination Kinetics Modelling Approaches for Estimating Bioconcentration Factors in the Freshwater Invertebrate, Gammarus Pulex. Sci. Total Environ. 2016, 547, 396-404.

(33) Karlsson, M. V.; Carter, L. J.; Agatz, A.; Boxall, A. B. A. Novel Approach for Characterizing PH-Dependent Uptake of Ionizable Chemicals in Aquatic Organisms. Environ. Sci. Technol. 2017, 51 (12), 6965-6971.

(34) Memmert, U.; Peither, A.; Burri, R.; Weber, K.; Schmidt, T.; Sumpter, J. P.; Hartmann, A. Diclofenac: New Data on Chronic Toxicity and Bioconcentration in Fish. Environ. Toxicol. Chem. 2013, 32 (2), 442-452.

(35) Malinowska, J. M.; Viant, M. R. Confidence in Metabolite Identification Dictates the Applicability of Metabolomics to Regulatory Toxicology. Curr. Opin. Toxicol. 2019, 16, 32-38.

(36) Sotto, R. B. D.; Medriano, C. D.; Cho, Y.; Kim, H.; Chung, I. Y.; Seok, K. S.; Song, K. G.; Hong, S. W.; Park, Y.; Kim, S. Sub-Lethal Pharmaceutical Hazard Tracking in Adult Zebrafish Using Untargeted LC-MS Environmental Metabolomics. J. Hazard. Mater. 2017, 339, $63-72$.

(37) David, A.; Lange, A.; Abdul-Sada, A.; Tyler, C. R.; Hill, E. M. Disruption of the Prostaglandin Metabolome and Characterization of the Pharmaceutical Exposome in Fish Exposed to Wastewater Treatment Works Effluent As Revealed by Nanoflow-Nanospray Mass Spectrometry-Based Metabolomics. Environ. Sci. Technol. 2017, $51(1), 616-624$.

(38) Taylor, N. S.; Gavin, A.; Viant, M. R. Metabolomics Discovers Early-Response Metabolic Biomarkers That Can Predict Chronic Reproductive Fitness in Individual Daphnia Magna. Metabolites 2018, 8 (3). DOI: $10.3390 /$ metabo8030042.

(39) Saei, A. A.; Jabbaribar, F.; Fakhree, M. A. A.; Acree, W. E.; Jouyban, A. Solubility of Sodium Diclofenac in Binary Water + Alcohol Solvent Mixtures at $25^{\circ}$ C. J. Drug Delivery Sci. Technol. 2008, 18 (2), 149-151.

(40) Huntscha, S.; Singer, H. P.; McArdell, C. S.; Frank, C. E.; Hollender, J. Multiresidue Analysis of 88 Polar Organic Micropollutants in Ground, Surface and Wastewater Using Online MixedBed Multilayer Solid-Phase Extraction Coupled to High Performance Liquid Chromatography-Tandem Mass Spectrometry. J. Chromatogr. A 2012, 1268, 74-83.

(41) Fu, Q.; Rösch, A.; Fedrizzi, D.; Vignet, C.; Hollender, J. Bioaccumulation, Biotransformation and Synergistic Effects of Binary Fungicide Mixtures in Hyalella Azteca and Gammarus Pulex: How Different/Similar Are the Two Species? Environ. Sci. Technol. 2018, 52 (22), 13491-13500.

(42) Chong, J.; Wishart, D. S.; Xia, J. Using MetaboAnalyst 4.0 for Comprehensive and Integrative Metabolomics Data Analysis. Curr. Protoc. Bioinforma. 2019, 68 (1), 1-128.

(43) Szymańska, E.; Saccenti, E.; Smilde, A. K.; Westerhuis, J. A. Double-Check: Validation of Diagnostic Statistics for PLS-DA Models in Metabolomics Studies. Metabolomics 2012, 8, 3-16.

(44) Bezanson, J.; Edelman, A.; Karpinski, S.; Shah, V. B. Julia: A Fresh Approach to Numerical Computing. SIAM Rev. 2017, 59 (1), 65-98.

(45) Rackauckas, C.; Nie, Q. DifferentialEquations.Jl - A Performant and Feature-Rich Ecosystem for Solving Differential Equations in Julia. J. Open Res. Software 2017, 5. DOI: 10.5334/jors.151.

(46) Rösch, A.; Gottardi, M.; Vignet, C.; Cedergreen, N.; Hollender, J. Mechanistic Understanding of the Synergistic Potential of Azole Fungicides in the Aquatic Invertebrate Gammarus Pulex. Environ. Sci. Technol. 2017, 51 (21), 12784-12795.

(47) Raue, A.; Kreutz, C.; Maiwald, T.; Bachmann, J.; Schilling, M.; Klingmüller, U.; Timmer, J. Structural and Practical Identifiability 
Analysis of Partially Observed Dynamical Models by Exploiting the Profile Likelihood. Bioinformatics 2009, 25 (15), 1923-1929.

(48) Jeon, J.; Kurth, D.; Ashauer, R.; Hollender, J. Comparative Toxicokinetics of Organic Micropollutants in Freshwater Crustaceans. Environ. Sci. Technol. 2013, 47 (15), 8809-8817.

(49) Ashauer, R.; Hintermeister, A.; O’Connor, I.; Elumelu, M.; Hollender, J.; Escher, B. I. Significance of Xenobiotic Metabolism for Bioaccumulation Kinetics of Organic Chemicals in Gammarus Pulex. Environ. Sci. Technol. 2012, 46 (6), 3498-3508.

(50) Sarda, S.; Page, C.; Pickup, K.; Schulz-Utermoehl, T.; Wilson, I. Diclofenac Metabolism in the Mouse: Novel in Vivo Metabolites Identified by High Performance Liquid Chromatography Coupled to Linear Ion Trap Mass Spectrometry. Xenobiotica 2012, 42 (2), 179194.

(51) Stierlin, H.; Faigle, J. W.; Sallmann, A.; Kung, W.; Richter, W. J.; Kriemler, H.-P.; Alt, K. O.; Winkler, T. Biotransformation of Diclofenac Sodium (Voltaren ${ }^{\circledR}$ ) in Animals and in Man. Xenobiotica 1979, 9 (10), 601-610.

(52) Salze, G. P.; Davis, D. A. Taurine: A Critical Nutrient for Future Fish Feeds. Aquaculture 2015, 437, 215-229.

(53) Mehinto, A. C. Chapter 5 and 6-Impacts of the Human Pharamaceutical Diclofenac in the Aquatic Environment, 2009.

(54) Longo, N.; Frigeni, M.; Pasquali, M. Carnitine Transport and Fatty Acid Oxidation. Biochim. Biophys. Acta, Mol. Cell Res. 2016, 1863 (10), 2422-2435.

(55) Sepúlveda, M. S.; Ralston-Hooper, K. J.; Sanchez, B. C.; HopfJannasch, A.; Baker, S. D.; Diaz, N.; Adamec, J. Use of Proteomic and Metabolomic Techniques in Ecotoxicological Research. Gen. Appl. Syst. Toxicol. 2011. DOI: 10.1002/9780470744307.gat215.

(56) Tiwari, S.; Mishra, M.; Salemi, M. R.; Phinney, B. S.; Newens, J. L.; Gomes, A. V. Gender-Specific Changes in Energy Metabolism and Protein Degradation as Major Pathways Affected in Livers of Mice Treated with Ibuprofen. Sci. Rep. 2020, 10 (1), 1-17.

(57) Arlos, M. J.; Schürz, F.; Fu, Q.; Lauper, B. B.; Stamm, C.; Hollender, J. Coupling River Concentration Simulations with a Toxicokinetic Model Effectively Predicts the Internal Concentrations of Wastewater-Derived Micropollutants in Field Gammarids. Environ. Sci. Technol. 2020, 54 (3), 1710-1719.

(58) Rand-Weaver, M.; Margiotta-Casaluci, L.; Patel, A.; Panter, G. H.; Owen, S. F.; Sumpter, J. P. The Read-across Hypothesis and Environmental Risk Assessment of Pharmaceuticals. Environ. Sci. Technol. 2013, 47 (20), 11384-11395. 